\title{
El medio ambiente en los Planes de Desarrollo de Andalucía
}

J. Marcos Castro y Antonio Morillas*

\section{Introducción.}

La riqueza ambiental de la región andaluza resulta una realidad en la que se coincide en todos los foros de debate. También resultan evidentes las ganancias que supondrían la revalorización y potenciación de estos activos ambientales, dentro de una estrategia hacia la sostenibilidad. Sin embargo, no existe consenso acerca de las pautas que se han de producir para alcanzar una senda de desarrollo sostenible. En nuestra región, tradicionalmente los planes de desarrollo han tenido la finalidad última de promover las condiciones objetivas para el crecimiento económico, la distribución equitativa de la riqueza y el aumento de la calidad de vida. En términos generales, el desempleo y el resto de problemas estructurales y de competitividad de la economía andaluza han sido el leit motif de los mismos. Los últimos planes, sin embargo, incorporan un matiz cualitativo en el concepto de desarrollo, la sostenibilidad ambiental, integrandola como un objetivo más de sus políticas.

En el presente artículo, se analiza la evolución del concepto ambiental en la planificación socioeconómica andaluza por excelencia: los planes de desarrollo. Éstos juegan un papel importante, no sólo en la definición de la política económica del gobierno regional, sino, también, a la hora de determinar la estrategia aplicada de la intervención comunitaria en la región, con la aprobación de los Marcos Comunitarios de Apoyo y los subsiguientes Programas Operativos. La incorporación del objetivo de sostenibilidad ambiental en las estrategias comunitarias ha tenido un efecto directo en los Planes elaborados en Andalucía. Las interrelaciones con el resto de objetivos de política económica, así como el análisis de las principales estrategias abordadas para la reducción de los problemas ambientales son tratadas desde la perspectiva de la planificación. El reciente Plan de Medio Ambiente de

- Antonio Morillas es Profesor Titular de Economía Aplicada de la Universidad de Málaga. J. Marcos Castro es Profesor Ayudante de Economía Aplicada de la Universidad de Málaga.

${ }^{1}$ No en vano algo más del $17 \%$ de su territorio se encuentra protegido. 
Andalucía entronca con esta evolución, en un intento integrador de las perspectivas socioeconómicas y ambientalistas, en el que cambia ligeramente la importancia relativa otorgada, hasta entonces, a cada una de las ellas.

\section{El medio ambiente como objetivo económico. El nuevo paradigma ambiental.}

La consideración de los recursos naturales siempre ha estado presente en el análisis económico. Economistas clásicos como Smith, Malthus o Ricardo, defendían una primigenia idea de los recursos naturales como condicionantes del desarrollo y riqueza de los países. Otros economistas modernos, entre los que destacan Marshall y, fundamentalmente Mill, proponen modelos de crecimiento en los que se vislumbra una idea de sostenibilidad también ambiental (estado estacionario de Mill, último estadio de desarrollo de Rostow).

Sin embargo, la ortodoxia económica no ha asumido hasta fechas muy recientes la inclusión de los objetivos ambientales entre el elenco de fines macroeconómicos ${ }^{2}$. Esta evolución ha sido impulsada por una serie de hechos (crisis energéticas de los setenta, catástrofes nucleares, manifestación de las desigualdades entre los paises del primer y tercer mundo, agujero de ozono, etc. que han motivado el tránsito desde las visiones de crecimiento ilimitado de los años sesenta a las actuales de desarrollo sostenible.

Para iniciar cualquier comentario acerca del tránsito hacia el nuevo paradigma ambiental es ya referencia obligada la I Conferencia de Naciones Unidas sobre el Medio Humano celebrada en Estocolmo en 1972, donde se realizó la primera declaración global sobre las relaciones entre medio ambiente y desarrolto. Así, se cuestiona que el crecimiento económico sea un fin en si mismo, coincidiendo con las teorías del Club de Roma expuestas en "Los Límites del Crecimiento" (1972). La búsqueda de un modelo de desarrollo sostenible comienza a ser una prioridad de las políticas económicas.

Sin embargo, el hito considerado por lo general más importante, será la elaboración en 1987, por la Comisión Mundial de Naciones Unidas sobre Medio Ambiente y Desarrollo, del conocido como Informe Brundtland:

2Compárese la evolución seguida en los objetivos de los programas de actuación del Banco Mundial, la OCDE o la ONU. 
"Nuestro Futuro Común". En él se acuña como concepto y objetivo global, el desarrollo sostenible como "el desarrollo que satisface las necesidades de la generación presente sin comprometer la capacidad de las generaciones futuras para satisfacer sus propias necesidades".

Un enunciado de los objetivos del modelo se describe en el Informe del Club de Roma "Más allá de los límites del crecimiento"(1992), donde se plantea la necesidad de optar por un modelo de desarrollo sostenible que permita la mejora del bienestar y la calidad de vida, a la vez que la conservación y correcta gestión de los recursos naturales. Así, una "sociedad sostenible" estaría interesada en el desarrollo cualitativo, no en la expansión fisica. No estaría ni a favor ni en contra del crecimiento, más bien comenzaría a discriminar entre distintos tipos de crecimiento y objetivos de crecimiento. Estas ideas se plasman a su vez en los procesos de planificación de la Comunidad Europea.

La Conferencia de las Naciones Unidas de Río de Janeiro (1992) supuso la aceptación internacional del reto hacia la sostenibilidad. Para la integración entre medio ambiente y el desarrollo se implica a los principales organismos internacionales, gobiernos, científicos y organizaciones sociales. En la Declaración de Río se expresa el compromiso político de abordar soluciones globales al orden económico y ambiental mundial, bajo el lema "pensar global, actuar local". Así, en su Preámbulo queda constancia del propósito de alcanzar "acuerdos internacionales en los que se respeten los intereses de todos y se proteja la integridad del sistema ambiental y de desarrollo mundial". A fin de alcanzar el desarrollo sostenible "la protección del medio ambiente deberá constituir parte integrante del proceso de desarrollo y no podrá considerarse en forma aislada" para lo que "los Estados deberían reducir y eliminar los sistemas de producción y consumo insostenibles y fomentar políticas demográficas apropiadas".

\section{Tendencias del Entorno. Estrategias de la CE en la política de desarrollo sostenible.}

En la filosofia de la Comunidad Europea, el término "desarrollo sostenible" viene descrito por una serie de conceptos interrelacionados, que aparecen en el mismo Tratado de la unión europea. Así, se asigna a la Comunidad la misión de promover "un crecimiento sostenible y no inflacionista que respete el medio ambiente". Igualmente, en su artículo $130 \mathrm{~A}$, se determina que "A fin de promover un desarrollo armonioso del conjunto de la Comunidad, ésta 
desarrollará y proseguirá su acción encaminada a reforzar su cohesión económica y social. La Comunidad se propondrá, en particular, reducir las diferencias entre los niveles de desarrollo de las diversas regiones y el retraso de las regiones menos favorecidas, incluidas las zonas rurales".

Previamente, la CE ya había anticipado su preocupación por el logro de esta simbiosis señalándose en la Declaración del Consejo de Europa de 1985 las relaciones recíprocamente beneficiosas entre economía y medio ambiente en lo que se avanza con el Cuarto Programa de Acción 1987-92, que ya recoge la proposición de la vinculación de la dimensión ambiental con las políticas que se proponen crear las condiciones necesarias para un crecimiento económico sostenido.

Asimismo, se plantea en el Tratado de Maastrich que "las exigencias de la protección del medio ambiente deberán integrarse en la definición y en la realización de las demás políticas de la Comunidad".

Con todo lo anterior, vemos como el "desarrollo armonioso", la "cohesión económico-social", el "uso racional de los recursos" y la "integración del medio ambiente en el resto de políticas" responden a esta filosofia hacia la sostenibilidad.

De forma más explícita, el desarrollo sostenible es objetivo central en la política comunitaria, como así se plasma en el título y contenido del Quinto Programa Comunitario de política y actuación en materia de medio ambiente y desarrollo sostenible (1992), "hacia un desarrollo sostenible", a fin de hacer confluir en una misma dirección las estrategias económicas y ambientales.

En el Quinto Programa, cuyo período de vigencia, flexible y abierto, es 1993-1997, se recoge una estrategia general de gestión integrada para proporcionar un marco medioambiental coherente para tipos de desarrollo integrado y sostenible.

Para la consecución de las estrategias contenidas en el Quinto Programa, se desarrollan una serie de instrumentos de política ambiental, muchos de los cuales suponen auténticas innovaciones normativas. Basándose en un enfoque integral del binomio desarrollo-medio ambiente, desde el que se comprendan las interacciones existentes entre ambas variables, se basa la actuación en la inclusión de la variable ambiental en el desarrollo económico y las políticas sectoriales, pasando a ser una componente endógena 
del proceso y, por tanto, sujeta a las reglas del mercado y con unos precios o costes a ser asumidos por los agentes.

Otro documento en el que, desde una visión más economicista e integrando el medio ambiente y el desarrollo, se definen las estrategias hacia la sostenibilidad es el "Libro Blanco sobre Crecimiento, Competitividad y Empleo" (1994). Este Informe es una referencia muy importante para establecer los objetivos de la planificación económica. En el mismo se aplica el análisis estratégico partiendo de un diagnóstico sobre el actual modelo de desarrollo comunitario, en el que la principal conclusión radica en la actual combinación subóptima de dos de los principales recursos comunitarios: la mano de obra y los recursos naturales.

El modelo actual se caracteriza pues por una utilización insuficiente del factor trabajo y una sobreutilización de los recursos naturales. La Comunidad se plantea pues como objetivo central la consecución de un crecimiento económico sostenible que contribuya a una mayor intensidad de empleo y a un menor consumo de energía y recursos naturales.

La sobreutilización de los recursos naturales, provocada gracias a que los precios de mercado no incorporan suficientemente su carácter limitado ni la consiguiente mayor escasez que supone su consumo. La menor cantidad y calidad de los recursos naturales representa una carga para las generaciones futuras y un menor potencial de prosperidad económica a largo plazo. Además, ciertas formas de contaminación amenazan no solamente a determinados sistemas ecológicos, sino el equilibrio natural de todo el planeta (cambio climático, capa de ozono, biodiversidad).

Como conclusión, parece clara la necesidad de un nuevo modelo de desarrollo sostenible, basado en unas políticas más adecuadas que ofrezcan a la sociedad un mayor grado de bienestar con un menor consumo y, consecuentemente, con una menor presión sobre los recursos naturales. En este contexto, el Libro Blanco aboga por la creación de puestos de trabajo que respondan a los nuevos retos ambientales, así como el aprovechamiento de los recursos humanos, los cuales deben realizarse a nivel local, fomentando la responsabilidad individual y la participación social. Por consiguiente, el nuevo modelo de desarrollo propugnado por la Comunidad combate la utilización ineficiente de los recursos disponibles desde una amplia perspectiva, esto es, tomando en consideración la calidad de vida de los ciudadanos. Todo ello en línea con el 
lema del Consejo Europeo de Birmingham (1992): "Una comunidad cercana a los ciudadanos".

De todo lo anterior, resulta meridianamente claro el concepto de desarrollo sostenible enunciado en las estrategias comunitarias e internacionales, desde la definición intuitiva dada por el Informe Brundtland hasta la concreción del Quinto Programa de Medio Ambiente y el Libro Blanco sobre Crecimiento, Competitividad y Empleo.

Sintetizando demasiado, podríamos concretar el modelo general como aquel desarrollo sostenible en el que se consigue un crecimiento equilibrado en el territorio (Cohesión Económica y Social en terminología comunitaria) y sostenible en el tiempo y el espacio, con unas consideraciones medio ambientales y de calidad de vida implícitos en todo el proceso.

\section{Los Planes de Desarrollo en Andalucía y el medio ambiente.}

El Estatuto de Autonomía de Andalucía (1983), en su título primero, concedió a la comunidad andaluza competencias en materia de política económica, social y territorial. La toma en consideración del medio ambiente, muy escasa en un principio, ha ido aumentando en los diferentes documentos sobre planificación del desarrollo realizados por el gobierno andaluz. Esta evolución puede resumirse como sigue:

a) Actuaciones anteriores al PEA84-86, contenidas en los planes de desarrollo, así como en las políticas sectoriales y territoriales que provenían del Estado Central. El medio ambiente no se recogía como una variable (instrumental $u$ objetivo) a tener en cuenta. Destacan dos recursos ambientales (el agua y la energía) que dan lugar a dos importantes estrangulamientos que se enfocan no desde una concep-

${ }^{3}$. Así entre los programas de inversión en obras de infraestructuras se encontraban objetivos ambientales. En un primer momento con las infraestructuras hidraúlicas, ante la necesidad de un desarrollo agrario en Andalucía, lo que obligó a la asunción de los elevados costes para abastecer los consumos agrarios intensivos en este factor. Por otra parte, las sucesivas crisis enérgeticas consigue la inclusión del abastecimiento y ahorro energético dentro de las actuaciones territoriales, aunque de nuevo, sin ninguna justificación ambiental más que de estrategia económica. 
ción de la sostenibilidad ambiental, sino económica ${ }^{3}$. El crecimiento se entiende a partir de una inversión pública dinamizadora de la actividad y el empleo.

b) Actuaciones incorporadas en el PEA84-86 y PADE87-90. Bajo la perspectiva del desarrollo endógeno como paradigma, en esta etapa los recursos naturales, si bien son admitidos como variables, constituyen restricciones exógenas al crecimiento. Su consideración aparece ligada a la ordenación del territorio y a los niveles de equipamientos, con objeto de procurar una mejor calidad de vida de la población.

c) PADE91-94 y orientaciones de los documentos previos del PEAHorizonte2000. Se producen los primeros avances en el proceso de endogenización de la variable ambiental, considerándose como uno de los factores productivos y endógenos (política de factores), así como uno de los condicionantes para que el modelo de crecimiento sea sostenible espacial y temporalmente. Aparecen conceptos como el de desarrollo sostenible, sinergia y ecodesarrollo. Se concede mayor importancia a los aspectos de formación, educación, cooperación e investigación ambientales.

Los factores que podrían explicar esta evolución del concepto medio ambiente en cada una de las fases anteriores, pueden ser los siguientes:

a) avance en las competencias. El margen de maniobra de las políticas socioeconómicas y de los subsiguientes ejercicios de planificación, ha dependido en cada caso de las condiciones competenciales y presupuestarias en las que se ha encontrado la Junta de Andalucía en cada momento.

b) planificación como proceso. El grado de madurez y la validación oficial del proceso de planificación como instrumento útil en la política sectorial y de desarrollo, han marcado, igualmente, el grado de definición y la ampliación de los objetivos de política económica regional en cada período. 


\section{Cuadro I}

Síntesis de las tendencias del entorno en la planificación ambiental en Andalucía

\begin{tabular}{|c|c|}
\hline Tendencias del entorno & \\
\hline Comunidad Europea & España \\
\hline $\begin{array}{l}\text { T. Masstrich: } \\
\text { - Desarrollo armonioso } \\
\text { - Cohesión económico-social } \\
\text { - La conservación, la protección y la mejora de la calidad } \\
\text { del medio ambiente } \\
\text { - Uso racional de los recursos } \\
\text { - Integración del medio ambiente en el resto de políticas } \\
\text { Libro Blanco Crecimiento: } \\
\text { - Mayor intensidad de empleo } \\
\text { - Uso eficiente de los recursos naturales y la energia } \\
\text { - Desarrollo de tecnologias limpias } \\
\text { V Programa Marco: } \\
\text { - Desarrollo sostenible } \\
\text { - Gestión racional de los recursos. (la calidad de la atmósfera, } \\
\text { la gestión de los recursos hídricos, la calidad del suelo, } \\
\text { la seguridad y rendimientos energéticos) } \\
\text { - Gestión del Transporte. } \\
\text { - Sectores de producción y servicios. } \\
\text { - Información, educación y formación. } \\
\text { - Evaluación y valoración } \\
\text { Investigación. }\end{array}$ & $\begin{array}{l}\text { Estrategia Nacional: } \\
\text { - Desarrollo } \\
\text { sostenible } \\
\text { - Uso racional de los } \\
\text { recursos naturales } \\
\text { - Protección del entor- } \\
\text { no } \\
\text { - Creación de empleo } \\
\text { ligado al medio } \\
\text { ambiente } \\
\text { - Lucha contra el } \\
\text { avance de la deserti- } \\
\text { ficación } \\
\text { - Calidad de las aguas } \\
\text { y optimización de los } \\
\text { usos } \\
\text { - Gestión de residuos } \\
\text { peligrosos } \\
\text { - Calidad del medio } \\
\text { ambiente urbano } \\
\text { - Red de información } \\
\text { y vigilancia sobre } \\
\text { aspectos ambienta- } \\
\text { les } \\
\text { Coordinación entre } \\
\text { administraciones. } \\
\text { Papel indicativo de } \\
\text { la administración } \\
\text { central. }\end{array}$ \\
\hline
\end{tabular}


Y, sobre todo, c) las tendencias del entorno, resumidas en el Cuadro 1. El acervo legislativo comunitario concede cada vez mayor importancia a la inclusión del medio ambiente en las políticas sectoriales y en el resto de las horizontales, así como al enfoque regional y local. El apoyo de estos argumentos, con importantes recursos e instrumentos financieros ad hoc. junto a la clasificación de Andalucía como región objetivo número 1 de la Comunidad Europea, hacen que este factor resulte, sin duda, de gran trascendencia para explicar la adopción de este punto de vista por parte del gobierno andaluz 4 .

Otro factor importante, si bien más difícil de valorar, es el entorno social, con aspectos tales como la toma de conciencia por el respeto al medio, los movimientos ecologistas, intelectuales, etc. Todo ello ha generado una actitud social más favorable hacia otros objetivos distintos a los iniciales de crecimiento económico, empleo y equipamiento e infraestructuras. Las políticas medio ambientales, así como otras relacionadas con los recursos humanos y tecnológicos, han podido verse beneficiadas por este hecho.

A continuación, realizaremos unos comentarios más amplios sobre cada uno de los planes económicos a los que hemos hecho referencia anteriormente. Después de analizar las metas u objetivos finales, nos centraremos en la presencia de los programas y acciones relacionadas con los recursos naturales. Una síntesis de los mismos puede verse en los Cuadros 2 y 3 del Anexo.

\subsection{Plan Económico para Andalucia 1984-1986}

El interés se centra en los objetivos finales de reducción del paro y elevación de la tasa de actividad, desarrollo más equilibrado de los diferentes sectores productivos de Andalucía, y la distribución más justa de la renta y la riqueza en Andalucía. Todo ello en base a la obtención de un rítmo de crecimiento económico por encima al conseguido por la economía española, así como al equilibrio intersectorial y regional de la economía, y la reducción del déficit de infraestructuras y equipamientos colectivos existente. Como puede comprobarse, un enfoque marcadamente desarrollista, con algunas concesiones al equilibrio productivo y espacial.

- Máxime si tenemos en cuenta que para poder acceder a los fondos estructurales (destinados algunos a objetivos ambientales) es necesaria la existencia previa de Programas de Desarrollo Regional, los cuales lógicamente incorporan la variable ambiental entre sus prioridades. 
La referencia a los recursos naturales más clara la encontramos entre los problemas económicos de alcance estructural, donde se incluye el "deterioro de la base primaria y del medio ambiente", básicamente la "contaminación de aguas marinas, la tala del bosque mediterráneo y la presencia de industrias altamente contaminantes en zonas de elevado potencial turístico".

Entre los programas de actuación e inversiones públicas recogidos en el PEA84-86 para la consecución de los objetivos finales, se encuentra el programa IX que liga los aspectos referidos a "Ordenación del Territorio y Medio Ambiente". Incluye actuaciones de protección ambiental, con objeto de reducir los estrangulamientos ambientales producidos por las elevadas densidades industriales en puntos muy localizados. El término calidad de vida se menciona igualmente al referirse genéricamente a estos efectos perniciosos de la actividad económica. Sin embargo, estas medidas tienen una menor importancia que las referidas a la reforma agraria (en caso de conflicto de objetivos) y la articulación y comarcalización del territorio. El crecimiento equilibrado consiste en minimizar la polaridad territorial existente y el desplazamíento de los factores productivos.

El gran logro del PEA, desde la perspectiva que nos ocupa, lo supone, probablemente, la inclusión en su programación de la creación de la Agencia de Medio Ambiente de Andalucía (1984), lo que hace que Andalucía sea una comunidad pionera en España en este tipo de Agencias. Otros logros que se plantean en este Plan son, fundamentalmente, la proyección de un ambicioso plan forestal (destinado a medidas de repoblación básicamente), los planes especiales de protección del medio físico y la red de espacios naturales protegidos.

En términos presupuestarios, los programas estrictamente medio ambientales abarcan el $2.60 \%$ de la inversión pública prevista para la Junta de Andalucía. El peso de la administración central en este programa resulta elevado, pues supone el $68,8 \%$ de la inversión total prevista para el mismo, agregando la inversión en medioambiente autonómica y central.

Dado el caracter horizontal de las políticas medioambientales, se da el caso en que otras medidas, normalmente encuadradas en los programas de agricultura y pesca, infraestructuras hidráulicas e $\mathrm{I}+\mathrm{D}$, aportan actuaciones relacionadas con la protección y valorización de los recursos naturales. En este caso, en el programa de agricultura se fija una inversión de 20.922,2 millones (de los que el Estado central aporta el 68,3\%), destinada a la repo- 
blación forestal y conservación del medio rural, medida relacionada de forma muy estrecha con la lucha contra la desertificación y los incendios.

\subsection{Programa Andaluz de Desarrollo Económico 1987-1990}

Ante la secularidad y gravedad de los problemas estructurales, de nuevo la creación de empleo y la distribución de las rentas y equipamientos sociales constituyen las metas de la programación socioeconómica andaluza de este cuatrienio.

El concepto de calidad de vida va asociado directamente a la consecución y distribución de la renta en el espacio andaluz, así como al acceso o disponibilidad de servicios y equipamientos sociales. La idea de potencial endógeno se aplica al capital humano y al fomento de $\mathrm{I}+\mathrm{D}$ de nuevas tecnologías, así como a la atracción de cabeceras de grandes empresas transregionales. Sin embargo, no se aplica esta definición al potencial ambiental o capacidad de desarrollo sostenible de determinados ecosistemas.

La estrategia territorial, que en el PEA anterior englobaba la acción sobre los recursos naturales, se basa en la ordenación del territorio para favorecer los efectos de difusión regional, definir las prioridades del sistema de incentivos y reducir los problemas de accesibilidad en el territorio andaluz. Los aspectos ambientales se ligan pues al territorio, con el objetivo último de la ordenación y vertebración del mismo.

Si bien en la definición de los objetivos y estrategias del PADE 87-90 no se menciona el término medio ambiente, entre los programas sectoriales dedicados al Área de Agricultura y Pesca sí aparecen objetivos relacionados con el mismo, tales como:

- Lucha contra la desertificación y erosión,

- Regeneración de pastizales.

- Adecuación de bosques y parques para ocio y esparcimiento.

- Prevención y lucha contra incendios.

- Desarrollo de zonas deprimidas.

- Regulación del balance hídrico.

Los programas específicos de "Protección del medio ambiente" suponen 10.936 millones (en ptas. de 1987), el 1,64\% del total de las inversiones de la administración autónoma para la financiación del PADE87-90. Las medidas 
TRABAJO. Revista Andaluza de Relaciones Laborales - n. ${ }^{2} 2 \cdot$ Diciembre 1996

básicamente van dirigidas hacia aspectos formativos y normativos del medio ambiente:

- Educación y formación ambiental

- Conservación y protección de la naturaleza

- Impacto ambiental

- Inventario y evaluación de recursos naturales

- Usos y actividades en el medio rural

- Apoyo a la iniciativa privada en la recuperación y mejora de la calidad ambiental

La aprobación del Plan Forestal Andaluz (en 1989, y con vigencia hasta el 2040) supone un importante paso en la política para la gestión racional de los recursos naturales, así como para el freno a los procesos de erosión y desertificación y la promoción del uso público forestal. Otros avances promovidos en el transcurso de este Programa son el Plan Director Territorial de Coordinación de Doñana, así como el Programa de Desarrollo Integrado para el Parque Natural de Cazorla-Segura-Las Villas.

\subsection{Plan Andauz de Desarrollo Económico 1991-1994}

EL PADE91-94 supone un cambio cualitativo respecto a los dos planes anteriores, que se manifiesta en la propia definición de las metas: "crecimiento sostenible y equilibrado y mejora de la calidad de vida y de la distribución de la riqueza". Manteniendo en un primer plano el predominio de los objetivos macroeconómicos, este Plan no sólo contempla la protección y revalorización ambiental con objeto de controlar la capacidad de carga del medio como destino de residuos, sino que adopta el concepto de capital ambiental como uno más de los factores productivos endógenos de la región, y determinante en usos como el turístico y el agrícola, tan importantes en Andalucía.

En corcondancia con las tendencias expuestas a nivel internacional, en este documento sobre planificación andaluza, el paradigma ambiental sustituye al paradigma desarrollista del crecimiento ilimitado, apareciendo conceptos como el de crecimiento sostenible ${ }^{5}$ o ecodesarrollo. Otro término de importante ubicación en el PADE91-94 es el de sinergia, que da cohesión a los quince objetivos finales (económicos, ambientales, territoriales y sociales).

\footnotetext{
${ }^{5}$ Que algo más tarde sería sustituido en la terminología ambientalista por el término desarrollo sostenible.
} 
Dentro del Grupo de programas referidos a la Política de Factores (junto a los recursos humanos, la I+D y el tejido empresarial), el relativo a los recursos naturales se centra en los siguientes aspectos:

- Gestión del Ecodesarrollo. Donde se concede importancia a la nueva conceptualización del crecimiento económico sostenible y a las necesidades de nueva infomación y nuevas pautas para sustentar este modelo.

- Superación de los déficit o estrangulamientos medioambientales de la estructura productiva andaluza, básicamente en tres áreas:

- gestión de residuos industriales, sólidos urbanos y agroganaderos.

- calidad de aguas y depuración de vertidos.

- protección a la atmósfera.

- La gestión de los espacios naturales protegidos, así como del impacto y dinamización socioeconómica del territorio circundante.

- Là ordenación de los recursos forestales, así como la restauración y protección del medio natural, luchando contra la desertización y los incendios, así como la racionalización del uso de los recursos hídricos y del suelo.

- La protección de la flora y la fauna, ampliando la cobertura legal actual, así como el seguimiento de su cumplimiento.

- La educación y formación ambiental; con especial hincapié en las generaciones más jóvenes, en las asociaciones ecologistas, en el medio urbano, así como en el empleo juvenil en ocupaciones relacionadas con la protección y recuperación del medio natural.

- La investigación ambiental, como elemento decisivo para desarrollar el potencial endógeno de nuestros recursos naturales y alcanzar una senda de crecimiento sostenible.

- La cooperación externa; ante el carácter global de los principales problemas ambientales, que requieren la cooperación entre regiones.

En este Plan se desarrolla formalmente un programa específico del medioambiente, el cual está dotado de cierta autonomía, es decir, en el PEA84-86, el medioambiente está agrupado con la ordenación del territorio, en el programa para mejorar la integración espacial de Andalucía; y en el PADE87-90, está encuadrado en los programas de infraestructura física y social, para alcanzar el nivel de equipamientos que aumente la calidad de vida. En el PADE91-94, ahora en el grupo de actividades productivas y recursos naturales, encontramos un completo conjunto de medidas que abarcan los principales aspectos de una política ambiental . 
Desde el punto de vista financiero, en este Plan se destina el 2,89\% de los recursos financieros totales al programa de medio ambiente, un total de $39.710,2$ millones (ptas. 1991). Si sólo tomamos en cuenta las operaciones de capital, este porcentaje asciende al $3,57 \%$.

\section{4. Plan Económico Andalucía Horizonte 2000}

En la actualidad se está elaborando el PEA 2000, que, desde una visión netamente estratégica, tras el intento fallido del gobierno minoritario socialista de sacar adelante el Plan de Desarrollo Andaluz, 1995-98, retoma la sucesión de planes de desarrollo económico de Andalucía hasta finales de siglo. En los borradores actuales se definen los objetivos y estrategias a seguir, así como un avance de las principales propuestas de actuación.

El objetivo final de la generación de empleo adquiere de nuevo el rango de prioritario frente al resto de objetivos, que sirven de plataforma para alcanzar el mismo. En este sentido, la consecución de un "modelo de desarrollo económico y social favorable al crecimiento del empleo" está en un segundo plano instrumental (son variables de progreso), donde la finalidad reside en la reducción efectiva de la tasa de paro en Andalucía.

Este modelo de desarrollo "solidario, equilibrado y sostenible" persigue en las dimensiones social, territorial y ambiental un modelo de desarrollo marcado por la generación de empleo, transformando las características estructurales del sistema regional andaluz y adaptándose a las tendencias del contexto exterior en base a tres retos estratégicos: la competitividad, la diversificación productiva y el desarrollo comercial.

En el PEA 2000 los recursos naturales se relacionan de forma muy directa con la "calidad de vida futura", así como con la "atracción de inversiones productivas de calidad, para crear condiciones favorables para la generación de empleo cualificado". Esta visión instrumental de los recursos naturales parece concretar el concepto primero de desarrollo sostenible, apoyándose en otros como el de calidad ambiental. Las necesidades de calidad ambiental, tanto por parte de la estructura productiva como de la propia sociedad andaluza, aparece, por tanto, como un objetivo implícito.

6.Argumento que puede ser considerado un pleonasmo, ya que los conceptos "solidario" y "equilibrado" estén contenidos en el de sostenible. 
De acuerdo con lo establecido en el Plan de Medio Ambiente, la erosión del suelo y la gestión del agua, se consideran los dos estrangulamientos ambientales más preocupantes, seguidos de la regeneración de ecosistemas, la eliminación de residuos, la agresión acústica, la contaminación atmosférica, fluvial y el deterioro del litoral.

Dentro del bloque de políticas específicas de desarrollo sostenible, en la política de medio ambiente se enuncian los objetivos y estrategias de la misma, que son los expuestos por el Plan de Medio Ambiente de Andalucía 1995-2000. Pensamos que vale la pena comentar brevemente los aspectos básicos del mismo, aunque no sea estrictamente un plan de desarrollo regional.

\section{El Plan de Medio Ambiente de Andalucía.}

El carácter horizontal de la política ambiental se ha puesto de manifiesto en los planes de desarrollo, y, de alguna forma, se ha explicitado la necesidad de la elaboración de un plan específico. El Plan de Medio Ambiente de Andalucía (PMA 1995/2000) supone un intento de dar respuesta a las necesidades existentes en Andalucía de un instrumento específico de planificación integrada, en el que, desde un prisma ambientalista explícito desde el diagnóstico inicial, se plantea un modelo de desarrollo sostenible. En este sentido, el concepto de sostenibilidad adquiere una importancia destacada en los aspectos socioeconómicos. El PMA pues, matiza las estrategias de desarrollo económico, de forma que resulten coherentes con una serie de metas a largo plazo que, independientemente de la naturaleza primera del plan (económica, ambiental, social), reflejan las aspiraciones últimas del proceso de planificación integrada: fomento del desarrollo sostenible y mejora de la calidad de vida.

Centrándonos en los aspectos referidos a crecimiento versus contaminación y uso de recursos naturales, el PMA, se plantea encarrilar la economía andaluza en una senda de desarrollo sostenible con la conservación de los recursos naturales, desde una triple perspectiva: promoviendo entre las diferentes actividades productivas un uso racional de los recursos naturales, favoreciendo la incorporación de las tecnologías ambientalmente más adecuadas y fomentando las nuevas actividades que están surgiendo en el ámbito de la industria relacionada con el medio ambiente. 
El PMA se plantea como objetivos los siguientes:

- Propiciar la mejora ambiental de las ciudades andaluzas, actuando sobre la calidad del aire, la contaminación acústica, el uso del agua, los residuos, el paisaje y los espacios naturales cercanos.

- Gestionar los recursos naturales en aras del desarrollo socioeconómico sostenible.

- Mejorar la calidad ambiental del litoral, recuperando las zonas degradadas y conservando el medio costero.

- Establecer un modelo hidrológico sostenible, apoyado en el uso eficiente del agua disponible y en una elevación notable de la calidad de la misma. Se trata de lograr un equilibrio sostenible entre la disponibilidad del recurso agua y su demanda, así como conservar la calidad de las aguas no contaminadas y mejorar la de aquellas que lo están.

- Fomentar las actividades económicas compatibles con el medio ambiente, constituyendo los aspectos ambientales el eje central de las actuaciones de desarrollo en el medio natural y en los diferentes sectores de la economía andaluza.

Para todo ello, el Plan formula una serie de ejes estratégicos, a los que asocia programas específicos de actuación:

- La dotación de equipamientos ambientales para la corrección de los déficits ambientales.

- La protección y conservación de la calidad ambiental mediante normativas, actuaciones y evaluaciones ambientales.

- La vigilancia e información, mediante equipamientos y sistemas de información.

- La consideración del medio ambiente como factor de desarrollo, en la triple dirección ya señalada de fomento de la potencialidad de los recursos naturales, creación de infraestructuras medioambientales y consolidación de un sector industrial específico.

- La participación ciudadana en la definición y consecución de un modelo de desarrollo sostenible.

- La coordinación interadministrativa en la planificación indicativa de las acciones y políticas.

- La formación y cualificación de los recursos humanos.

- La educación y comunicación de las conductas ambientalmente adecuadas y difusión de información ambiental.

- La cooperación internacional. 
Del análisis de la jerarquía de programas, los relativos a la gestión de los recursos hídricos y los destinados a reducir los procesos de erosión y desertificación son los que adquieren un mayor peso relativo en términos de aportación sinérgica al resto de actuaciones. Desde el punto de vista financiero, las partidas destinadas a la política forestal $(38,11 \%)$, a la reforestación de tierras agrarias y medidas agroambientales $(24,73 \%)$ y a la depuración de aguas residuales $(22,20 \%)$, corroboran la gran preocupación por los niveles de desertificación que se dan en determinadas zonas de la región.

El programa de medio ambiente urbano representa apenas el $6,06 \%$ del presupuesto destinado para el período de vigencia del Plan, sin embargo, por su novedad, se destaca sobre el resto de programas y actuaciones específicas, máxime si tenemos en cuenta que la mayor parte de la población reside en núcleos urbanos, los cuales manifiestan, sobre todo en las áreas metropolitanas, problemas de sostenibilidad importantes.

Como se señala en el análisis que realiza el PMA de la evolución del gasto ambiental en Andalucía, se produce una evolución del gasto acorde con el enfoque concreto de la política ambiental. En un primer momento se centra en programas de conservación, protección y restauración del patrimonio natural, para, conforme se consiguen logros en esta política y madura el proceso de planificación ambiental, ponderar más favorablemente otros objetivos relacionados con las consecuencias ambientales de los procesos productivos y la calidad ambiental (del agua, de la atmósfera, etc.), así como la formación y educación ambiental.

\section{Conclusiones.}

La inclusión de la variable ambiental en los procesos de planificación económica de Andalucía ha ido en creciente evolución desde la consecución de la Autonomía, lo cual ha ido acompañado de avances legislativos ${ }^{7}$ y de la redacción de planes específicos o sectoriales que contemplan la dimensión ambiental $^{s}$. Todo ello ha desembocado en la elaboración del Plan de Medio Ambiente de Andalucía como instrumento para la consecución de una senda de desarrollo sostenible para la región.

?. Como la Ley Forestal de Andalucia (1992), la Ley de Protección Ambiental (1994) y la Ley de Espacios Naturales Protegidos (1989).

'. Como el Plan Forestal de Andalucia (1989), el Plan Director de Infraestructuras (1994) el Plan Energético (1994), etc. 
Se ha producido una traslación de las tendencias del entorno al caso de Andalucía, al asumir el cambio producido desde el paradigma desarrollista hacia el llamado nuevo paradigma ambiental.

Si bien existe una continuidad en la evolución apuntada arriba, cada plan económico presenta algunas diferencias contextuales. Así, el PEA84-86 y el PADE 87- 90 están encardinados hacia el concepto de desarrollo endógeno, donde se determina como factor de competitividad el desarrollo del potencial endógeno de la región. El medio ambiente no se recoge explícitamente como uno de los factores endógenos, aunque sí es determinante para la consecución de unos estándares de calidad de vida. Se asocia pues al concepto de infraestructura y a la ordenación del territorio. Por otra parte, el PADE91-94 y el PEA2000, en elaboración, están imbuidos por la filosofía del desarrollo sostenible y equilibrado, asumiendo la necesidad de un cambio de orientación, integrándose el medio ambiente como uno de los factores determinantes de la sostenibilidad de los niveles de crecimiento. Los conceptos de sinergia y calidad ambiental caracterizan esta visión en la que se concede mucha importancia a la interacción entre el sistema productivo y los recursos naturales, así como a la cohesión interna entre los objetivos planteados.

En todos ellos, podemos observar una evolución de la política ambiental que en un primer momento se ocupa casi exclusivamente de la protección ambiental para pasar, en los últimos pasos, a ocuparse también de los aspectos formativos, la investigación aplicada y la cooperación. La distinción entre ámbitos de actuación (medio rural, medio urbano, medio forestal, litoral, etc.) supone también la adopción de una perspectiva más útil en estos casos.

Los últimos Planes parten de una visión estratégica en la que no se pretende plasmar fielmente todos y cada uno de los aspectos de la realidad ambiental y socioeconómica de la región, sino determinar los estrangulamientos estructurales y las características más importantes que permitan diseñar una estrategia de desarrollo más orientada a la acción que a la descripción. Las tendencias del entorno son asimismo examinadas desde esta perspectiva, concediéndose mucha atención a las pautas que se producen de "mercantilización" de los recursos naturales, así como el desarrollo del llamado negocio ambiental, apareciendo el sector productivo del medio ambiente.

La planificación se entiende como un proceso abierto, más que como un documento cerrado que caduca unos años más tarde, por lo que se concede una gran importancia a los mecanismos de adaptación a las tendencias del 
entorno, así como de control y evaluación del proceso para dotarlo de esa continuidad y flexibilidad necesarias en toda estrategia. Sin embargo, destaca el hecho de que ningún plan, en su documento específico, ha realizado nunca una crítica constructiva a los resultados del anterior plan, partiendo de una situación de "tabla rasa" en la que se vuelven a diagnosticar los problemas y la caracterización del crecimiento y la estructura de la región. En muchos casos, como hemos tenido la oportunidad de comprobar (véanse los cuadros del Anexo), coinciden enteramente las metas finales (lo cual es lógico), y en varios casos, coinciden los objetivos intermedios y los instrumentales. Esto indica que el diagnóstico que se realiza de la realidad andaluza, pone de manifiesto la permanencia en el tiempo de los mismos problemas y que los medios a los que se recurre son básicamente los mismos, lo cuál es la crítica más terrible que se puede realizar al éxito real de los propios planes. Por otro lado, si bien en el enunciado de los objetivos medio ambientales se pretenden cambios èn las estructuras productivas y en la orientación de las políticas sectoriales, así como nuevos esfuerzos específicos de protección y revalorización de los recursos naturales, tales objetivos quedan en muchos casos difuminados, produciéndose discordancias entre lo planteado y lo realmente especificado en términos presupuestarios.

Resultaría dificil evaluar el grado de consecución de los objetivos. $\mathrm{Ni}$ siquiera los propios documentos, a pesar del seguimiento obligatorio que ha de realizarse en el período de vigencia de un plan, recogen, como se ha dicho anteriormente, tal tipo de análisis sobre los inmediatamente anteriores. El hecho de que se trate, básicamente, de incidir en objetivos genéricos, tales como la estructura socioeconómica y ambiental de la región, resolviendo los problemas estructurales ${ }^{9}$, más que, por ejemplo, en alcanzar alzas coyunturales de las tasas de empleo y producción o de reducción del consumo depredador de recursos naturales, hace más difícil aún la valoración pertinente. El análisis de la eficacia y eficiencia del proceso de planificación del desarrollo, con el establecimiento de indicadores de seguimiento y evaluación, se ha tenido en cuenta, al menos sobre el papel, en casi todos los planes. Sin embargo, insistimos, no se ha recogido, a posteriori, en forma suficientemente clara, tal valoración en los documentos de los planes sucesivos. De cualquier forma, si aceptamos la globalidad de los objetivos, debería darse como válido, asimismo, un análisis respecto al control de su consecución de la misma dimensión.

${ }^{9}$ Que en el tema ambiental son básicamente problemas de desertificación y erosión, la protección de los ecosistemas, asi como el tratamiento de los residuos industriales, agrarios y urbanos. 
En este sentido, paraconcluir, hay que subrayar que diversos estudios ${ }^{10}$ han puesto de manifiesto el fracaso real de los objetivos básicos de la planificación, tanto en términos económicos (paro, mayor especialización productiva) como medio ambientales (mayor dependencia de los recursos naturales de la región, desviándose, así, de la senda de un desarrollo sostenible).

10 Véase, por ejemplo, M. DELGADO (1990 y 1995). A. MORILLAS (1995), A. MORILLAS; E. MELCHOR y J.M. CASTRO (1996) o el propio diagnóstico del PMA, en su capítulo 3. 


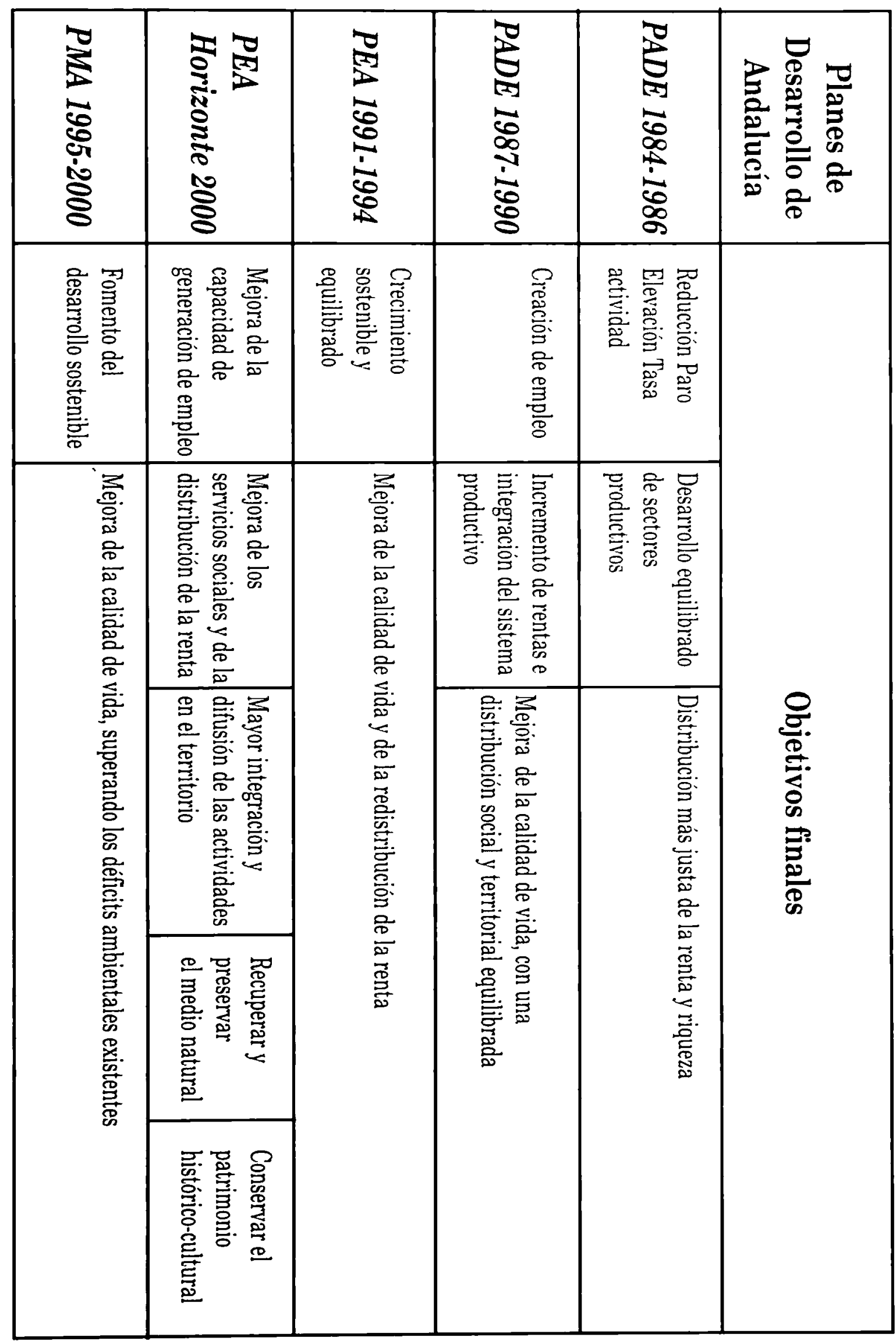




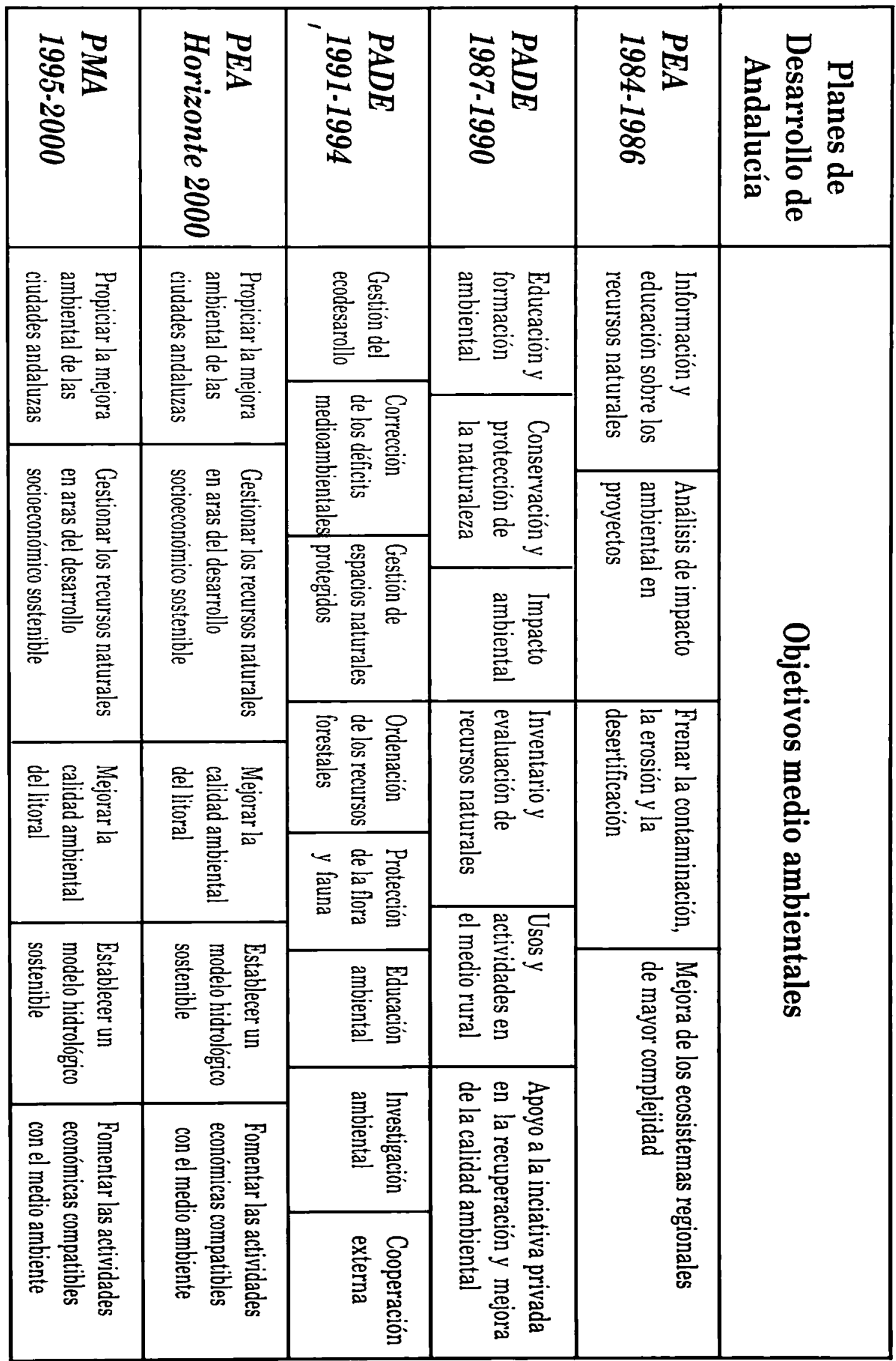




\section{Bibliografía}

Consejería de Economia y Hacienda: Plan Económico para Andalucía, 1984-86. Consejeria de Economia y Hacienda: Programa Andaluz de Desarrollo Económico, 1987-90.

Consejería de Economia y Hacienda: Programa Andaluz de Desarrollo Económico, 1991-94.

Consejeria de Economia y Hacienda: Plan Económico - Andalucia Eoronte 2000 (documentos previos).

Consejería de Medio Ambiente: Plan de Medio Ambiente de Andalucia, 1995. 2000.

Delgado Cabeza, M.(1990): Condicionantes del futuro económico de Andalucia. Revista de Estudios Regionales, núm 90; pp 25-55

Delgado CABEzA, M. (1995): Las relaciones con el exterior de la estructura productiva andaluza. Análisis de las necesidades de importación. 19801990. Contabilidad Regional y Tablas Input-Output de Andalucía. Análisis de resultados. Vol I, pp 272-315

Hall, P. (1993): Urban and Regional Planning. Allen \& Unwin. 2nd. Edition 1993. London

Morillas Raya, A. (1995): Aplicación de la teoría de grafos al estudio de los cambios en las relaciones intersectoriales de la economía andaluza en la década de los 80. Contabilidad Regional y Tablas Input-Output de Andalucía. Análisis de resultados. Vol I, pp. 90-140

Morillas, A.; MElChOR, E.; CASTRO, JM.(1996): Crecimiento y medio ambiente en Andalucia. Revista de Estudios Regionales, núm. 46 (en prensa). 